\title{
Polychromatic CT Data Improvement with One-Parameter Power Correction
}

\author{
Anastasia S. Ingacheva $\mathbb{D}^{1}$ and Marina V. Chukalina $\mathbb{D}^{1,2}$ \\ ${ }^{1}$ Institute for Information Transmission Problems RAS, Moscow, 127051, Russia \\ ${ }^{2}$ FSRC "Crystallography and Photonics" RAS, Moscow, 119333, Russia \\ Correspondence should be addressed to Marina V. Chukalina; chukalinamarina@gmail.com
}

Received 7 December 2018; Revised 13 February 2019; Accepted 13 March 2019; Published 11 April 2019

Academic Editor: Francisco J. Montáns

Copyright (C) 2019 Anastasia S. Ingacheva and Marina V. Chukalina. This is an open access article distributed under the Creative Commons Attribution License, which permits unrestricted use, distribution, and reproduction in any medium, provided the original work is properly cited.

\begin{abstract}
Standard approaches to tomography reconstruction of the projection data registered with polychromatic emission lead to the appearance of cupping artifacts and irrelevant lines between regions of strong absorption. The main reason for their appearance is the fact that part of the emission with low energy is being absorbed entirely by high absorbing objects. This fact is known as beam hardening $(\mathrm{BH})$. The procedure of processing projection data collected in polychromatic mode is presented; it reduces artifacts relevant to $\mathrm{BH}$ and does not require additional calibration experiments. The procedure consists of two steps: the first is to linearize the projection data with one-parameter power correction, and the second is to reconstruct the images from linearized data. Automatic parameter adjustment is the main advantage of the procedure. The optimization problem is formulated. The system flowchart is presented. The reconstruction with different powers of correction is considered to evaluate the quality reconstruction.
\end{abstract}

\section{Introduction}

$\mathrm{X}$-ray computed tomography $(\mathrm{CT})$ is a nondestructive technique for studying and visualizing interior features of an object without its physical destruction. Nowadays CT has become one of the main noninvasive diagnostic methods in medicine, scientific research, and industrial applications. $\mathrm{X}$-ray computed tomography in medicine is used for $3 \mathrm{D}$ visualization of the inner structure of patient's organs for studying diseases and their course controlling $[1,2]$. Dental tomography is used for planning implantation and prosthetics $[3,4]$. In industry, CT is used for quality control of products $[5,6]$. In progressive scientific research, computed tomography serves as the method for examination of pressure retaining nuclear plant components [7].

The task of CT consists of measuring projection data from multiple orientations of an object placed on a tomographic set-up and subsequent processing of the projection data in order to obtain a solution of the inverse tomography problem. Many tomography reconstruction algorithms are based on the assumption that attenuation of the X-rays in a material linearly depends on the length path of probing radiation.
The main, becoming classical, types of the algorithms are based on integral or algebraic approaches like Fourier Back Transform (FBP) or Algebraic Reconstruction Technique (ART) [8]. However, nowadays algorithm development work is in progress due to the fact that the objects with nonstandard attenuation properties [9] have to be measured or nonstandard experimental conditions are required (lowdose tomography) [10]. The works are focused also on an improvement of the algorithms properties like convergence $[11,12]$ or the quality of the reconstruction [13].

Projection data which satisfy linear model can be obtained by usage of monochromatic X-ray probe only. However, X-ray laboratory sources produce polychromatic beams; therefore, data collected in lab conditions are not linear. Classical reconstruction algorithms to the polychromatic data lead to different artifacts and distortions such as "cupping artifact" or artificial strips and lines between regions with strong absorption [14]. The main reason for their appearance is that the part of the radiation with low energy is fully absorbed by the regions containing high- $\mathrm{Z}$ materials. This effect is known as beam hardening (BH) and was described by Brooks and DiChiro [15]. The authors show 
that data nonlinearity in tomographic reconstruction can exceed $10 \%$ of average value, therefore noticeably limiting the usage of CT in tasks where we need to estimate not only the global structure but also the absorption value in every point. The suppressing of artifacts due to the polychromatic nature of the probing emission was the target for scientists for decades. As a result, nowadays there are a lot of methods for artifacts suppression. These methods can be generally divided into five groups: hardware filtering, usage of dual-energy scaling, preprocessing of projection data, reconstruction with iterative methods using the model of polychromatic radiation, and reconstruction result postprocessing.

The first methods group is based on hardware filtration. The idea is to use metal objects to partially absorb the low energy radiation. Material and shape of the filter are highly dependent on the material of anode, current, and peak voltage of the X-ray tube. The main target of researches in this group of methods is to incorporate new filters $[16,17]$. This hardware filtration reduces artifacts caused by beam hardening effects and also reduces the number of photons, which leads to bad signal/noise ratio and causes CT image degradation.

The second group includes methods for tomography setups with two X-ray tubes [18-20]. The usage of two energies allows obtaining two sets of the reconstruction results. If a device with two energies is used, then two reconstruction results are calculated: one for high energy and one for low energy. Thus, it is possible to select the image highly dependent on $\mathrm{Z}$ which gives us a clue on the sample material. However, this technique has its own drawbacks such as high complexity of realization and strong sensitivity to noise. Another drawback is that it is required to use $\mathrm{X}$-ray radiation twice for the object which increases the scanning time and radiation dose received by the sample.

The methods of the third group use preprocessing of the registered projection data until they transfer to reconstruction. The processing is based on the calibration curves usage. The test samples are measured to draw the calibration curves. First, [21] suggested the use of a polynomial function of power 2 and 3 with one parameter to approximate the curves. Then it was shown that if the sample has high absorbing inclusion, then a polynomial function of power eight or greater has to be used for approximation [22].

Next group consists of iterative methods including the correction procedure in the core of reconstruction [23-25]. These methods meet different handicaps and limitations such as requirements for knowledge about the sample or beam spectrum. But the main drawback of these methods is the time of reconstruction.

The methods of the last group propose to include the postprocessing of reconstructed data. Methods from this group do not change projection data and use classical reconstruction algorithms. They are focused on the removal of artifacts from the reconstructed images. One of these methods is based on the blind deconvolution method which is widely known in computer vision for correction of blurred images [26]. Another method from this group evaluates missing data using discrete cosine transform and antialiasing filter which preserves borders [27]. The main drawback of methods from this group is that all of them use image processing methods and do not take into account image origin. Therefore these methods can remove important information from the reconstructed image as well as adding nonexistent information to them.

In the paper, we present the procedure of processing projection data collected in the polychromatic mode which does not require additional calibration experiments and reduces artifacts relevant to $\mathrm{BH}$. The sections are organized as follows. After the optimization problem statement, we describe the software implementation. In the "Results" the proposed algorithm output is given. The verification of the obtained results is presented in the "Discussion". In our research, we used the projections, collected with the tomography set-up at FSRC "Crystallography and Photonics" RAS.

\section{Optimization Problem Statement}

If we use X-ray monochromatic radiation with initial intensity $I_{0}$, then signal $P$ collected by detector cell $s$ ( $S$ is the entire set of detector cells, and therefore $s \in S$ ) can be described with the law of Beer-Lambert as

$$
P(s, \alpha)=I_{0} \mathrm{e}^{-\int_{L} \mu(l) d l},
$$

where $\alpha$ is a rotation angle of the object and $L$ is the straight path of the probe from the source to the detector through the object ( $d l$ is a step). Two coordinates $(s, \alpha)$ fully specify the position of the probe with zero thickness and the probing direction. Before the reconstruction of the projection data, there is a linearization procedure:

$$
R(s, \alpha)=\ln \left(\frac{I_{0}}{P(s, \alpha)}\right)=\int_{L} \mu(l) d l .
$$

$R(s, \alpha)$ is an integral of function $\mu(l)$ along the direction $L$, which is orthogonal to vector $\overrightarrow{\mathrm{n}}=(\cos \alpha$, $\sin \alpha)$, so $R$ is function that describes Radon transform. The Radon transform data is often called a sinogram because the Radon transform of an off-center point source is a sinusoid. Most of the reconstruction algorithms work with sinograms. Let us consider total intensity of all detector cells for rotation angle $\alpha$ :

$$
T_{\alpha}=\sum_{s \in S} R(s, \alpha)
$$

$T_{\alpha}$ from (3) equals summation of the function $\mu(l)$ over the entire probing area since the position of the probing beam depends on coordinate $s$, which takes all values from its range. Therefore the value of $T_{\alpha}$ is constant for every $\alpha \in \mathrm{A}$, preserving the property of sinogram invariance relatively to the rotation angle. This is the property of Radon invariant.

However, in laboratory tomography scanners typically use X-ray tubes with polychromatic beam spectrum (i.e., wide energy spectrum from the range $\left.\left[0, E_{\text {max }}\right]\right)$. Equation (4) describes the value registered by the detector in this case:

$$
P(s, \alpha)=\int_{0}^{E_{\max }} I_{0}(E) \mathscr{X}(E) \mathrm{e}^{-\int_{L} \mu(E, l) d l} d E,
$$




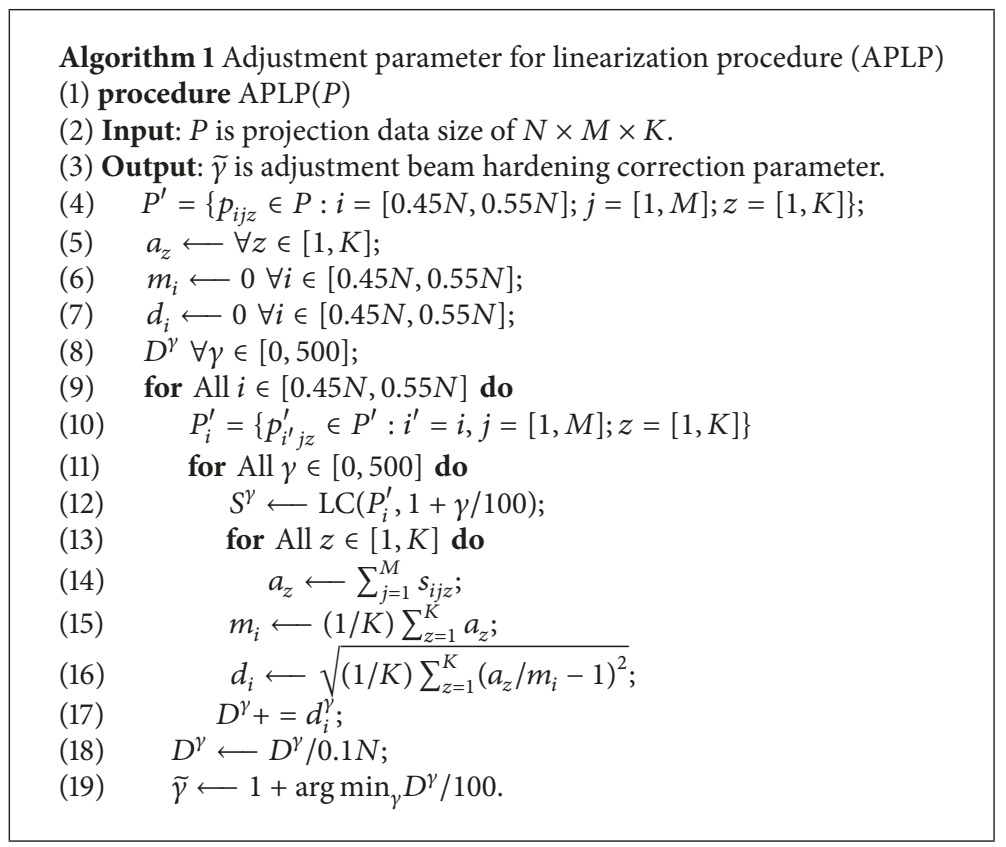

Pseudocode 1: Pseudocode of adjustment parameter for linearization procedure algorithm.

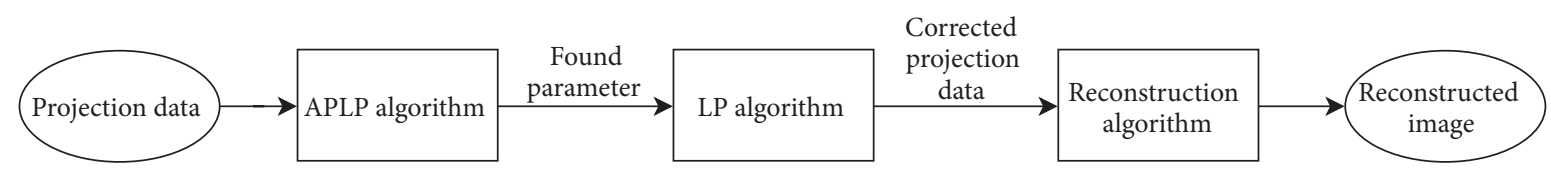

FIGURE 1: Flowchart of our proposed processing method for polychromatic data.

where $\mathscr{X}(E)$ is a sensitivity function of the detector. Therefore, (1) has been changed with additional integral through energies. Since the local absorption function of the object $\mu(E, l)$ also depends on energy $E$, the data registered in polychromatic mode cannot be represented as exact Radon transform of any object. Usage of the standard reconstruction algorithms on the data of this type leads to the appearance of the different artifacts. For decreasing the artifacts one has to find the transformation which will linearize data.

$$
f(P(s, \alpha))=-\int_{L} \mu(l) d l
$$

We use one power approximation [28].

$$
f=\left(\ln \left(\frac{\int_{0}^{E_{\max }} I_{0}(E) \mathscr{X}(E) d E}{P(s, \alpha)}\right)\right)^{\gamma}
$$

$\int_{0}^{E_{\max }} I_{0}(E) \mathscr{X}(E) d E$ is easily estimated from the values in the "empty" detector cells containing nonattenuated initial beam.

If the power value $\gamma$ is evaluated using the property of Radon invariant preserving, then the optimization problem is formulated as

$$
\sqrt{\frac{1}{\mathrm{~A}} \sum_{\alpha \in \mathrm{A}}\left(\frac{\sum_{s \in S} f(P(s, \alpha))}{M\left[\sum_{s \in S} f(P(s, \alpha))\right]}-1\right)^{2}} \underset{f}{\longrightarrow} \min ,
$$

where $M[\cdot]$ is an operator of a mean value.

\section{Software Implementation}

Figure 1 shows the flowchart of our proposed method of polychromatic data processing.

The suggested method consists of three steps: with the first step, we find optimal parameter value with adjustment parameter for linearization procedure (APLP) algorithm. With the second step, we correct polychromatic data to make it closer to linear model with linearization procedure (LP) algorithm. After the projection data is corrected, the reconstruction algorithm is executed as the third step. The detailed discussion of each of these three algorithms is provided below.

To find optimal parameter value we execute APLP algorithm. The APLP algorithm takes projection data $P$ as an input. Projection data have three dimensions $(N \times M \times K): N$ is the number of detector rows, $M$ is the number of detector columns, $K$ is the number of rotation angles. Pseudocode of this procedure is shown in Pseudocode 1.

On the initial step of the algorithm, we select part of the projection data $P^{\prime}$ which is within 5\% distance range from the central projection. If our tomography task is twodimensional, then the algorithm is executed for all projection data (one line). We search the parameter value $\tilde{\gamma}$ in the range $[1.00 ; 6.00]$ with step equal to 0.01 . Every line from the part of the projection data $P^{\prime}$ is being corrected with current power value $1+\gamma / 100$. For this we used LP algorithm, which will 

Algorithm 2 Linearization procedure (LP)
(1) procedure $\operatorname{LP}(P, \gamma)$
(2) Input: $P$ is projection data size of $N \times M \times K$,
(3) $\quad \gamma$ is beam hardening correction factor.
(4) Output: $S$ is corrected sinogram size of $N \times M \times K$.
(5) for All $p_{i j z} \in P$ do
(6) $\quad \widetilde{p}_{i j z} \longleftarrow-\ln p_{i j z}$;
(7) $\quad s_{i j z} \longleftarrow\left(\widetilde{p}_{i j z}\right)^{\gamma}$.

Pseudocode 2: Pseudocode of linearization correction algorithm.

be described in the next section. Then we evaluate Radon invariant for every angle $m_{i}$, average Radon invariant, and the root-mean-square of the ratio between Radon invariant for every angle $d_{i}$ and average Radon invariant $m_{i}$. Optimal value is found using brute force search over the range $D^{\gamma}$. We use brute force search since there are no obvious reasons to think that dependence between the value and the Radon invariant is differentiable and has only one minimum. As a result of the APLP algorithm, we obtain an optimal parameter for correction projection data.

LP algorithm is used for polychromatic projection data correction. Pseudocode of LP algorithm is shown in Pseudocode 2. Input parameters for the LP algorithm are polychromatic projection data $P$ and the parameter $\gamma$ (power value for data correction). Each pixel of the projection data is corrected independently from the others; i.e., correction is performed pixel by pixel.

We used the FBP algorithm for data reconstruction since it is well studied and widely used in both medical and industrial CT scanners. It can be noticed that the suggested polychromatic data correction allows usage of any reconstruction algorithm which works in the paradigm of linearized data. The suggested method will complement well the modification reconstruction algorithms focused on suppressing another type of artifacts, for example, artifacts arising from disturbances in the geometry of the CT set-up or the presence of high-level noise.

\section{Results}

Linear dimensions of sample are $7 \times 7 \times 12 \mathrm{~mm}$. For data acquisition, we used the micro tomograph which was assembled in the "Crystallography and Photonics" Federal Research Center of the Russian Academy of Science [29]. We used an X-ray tube with Mo anode as a radiation source and a vacuum gauge for intensity stability reasons. The projections were collected with the XIMEA-xiRay 11 Mpix detector. The measurement mode parameters for the tube were $40 \mathrm{kV}$ voltage, $20 \mathrm{~mA}$ current, and $5 \mathrm{~s}$ acquisition time per frame. A source to object distance was $1.2 \mathrm{~m}$ and object to detector distance was $0.05 \mathrm{~m}$. Every rotational scan consisted of 400 projections with an angular constant step size of $0.5 \mathrm{deg}$. The detector pixel resolution was $9 \mu \mathrm{m}$. The geometrical configuration of the experiment is such that the X-ray beam can be considered as parallel since its angle of divergence
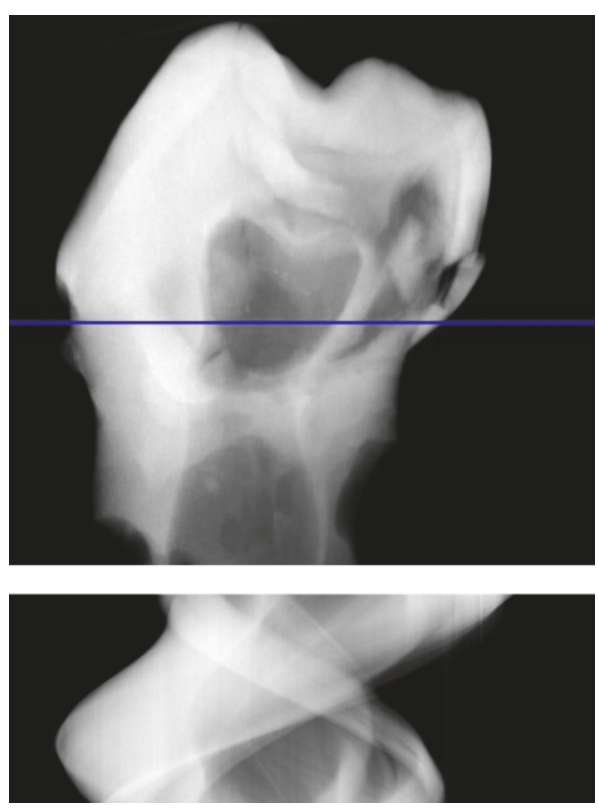

Figure 2: Sample projection (top) and sinogram (bottom).

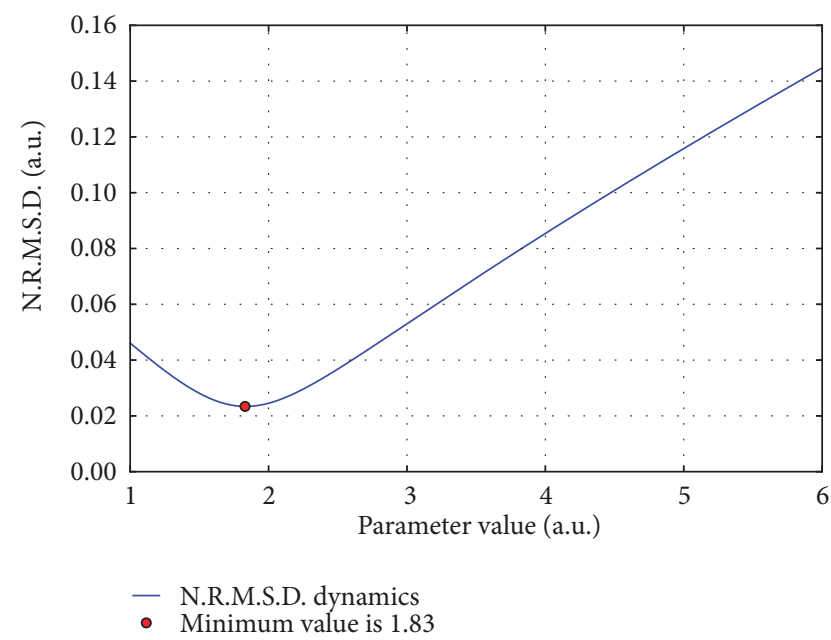

FIGURE 3: NRMSD dynamics depending on the parameter value.

is less than 1 deg. [30]. No filter or other optics was used to produce a monochromatic beam, so the work mode was polychromatic.

A CT projection of the sample is shown in the top of Figure 2. The blue line indicates the cross-sections chosen for the $2 \mathrm{D}$ reconstruction. The sinogram pertaining to the crosssections is shown at the bottom of Figure 2.

We applied the method of polychromatic data processing described above to the registered data. Figure 3 shows the behavior of criteria for different correction parameter values. It can be seen in Figure 3 that the optimal value of the parameter is 1.83 . The found value agrees with the world publications on this topic, which suggest using the value 2.0 when weak absorption coefficient inclusions are presented in an investigated object and raising the value in case of presence 


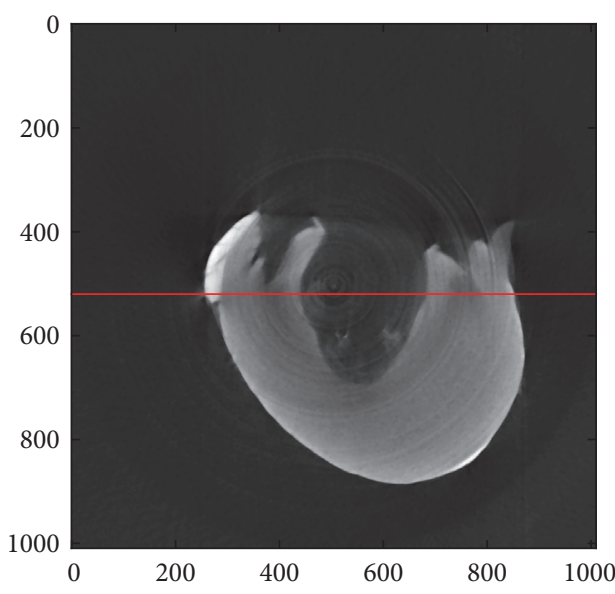

(a)

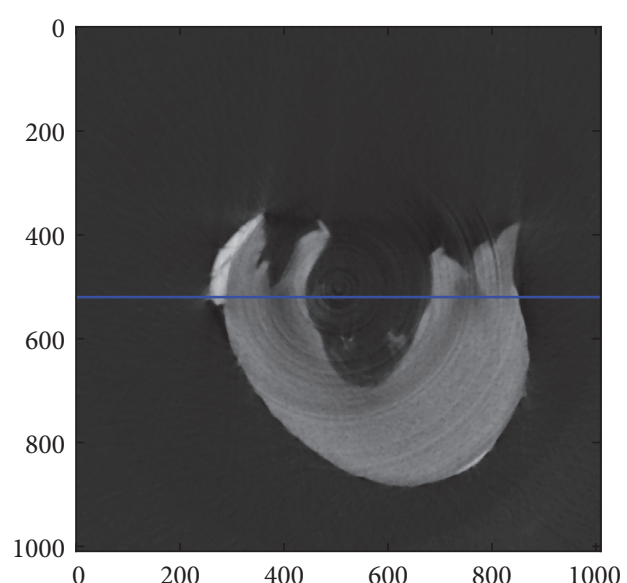

(b)

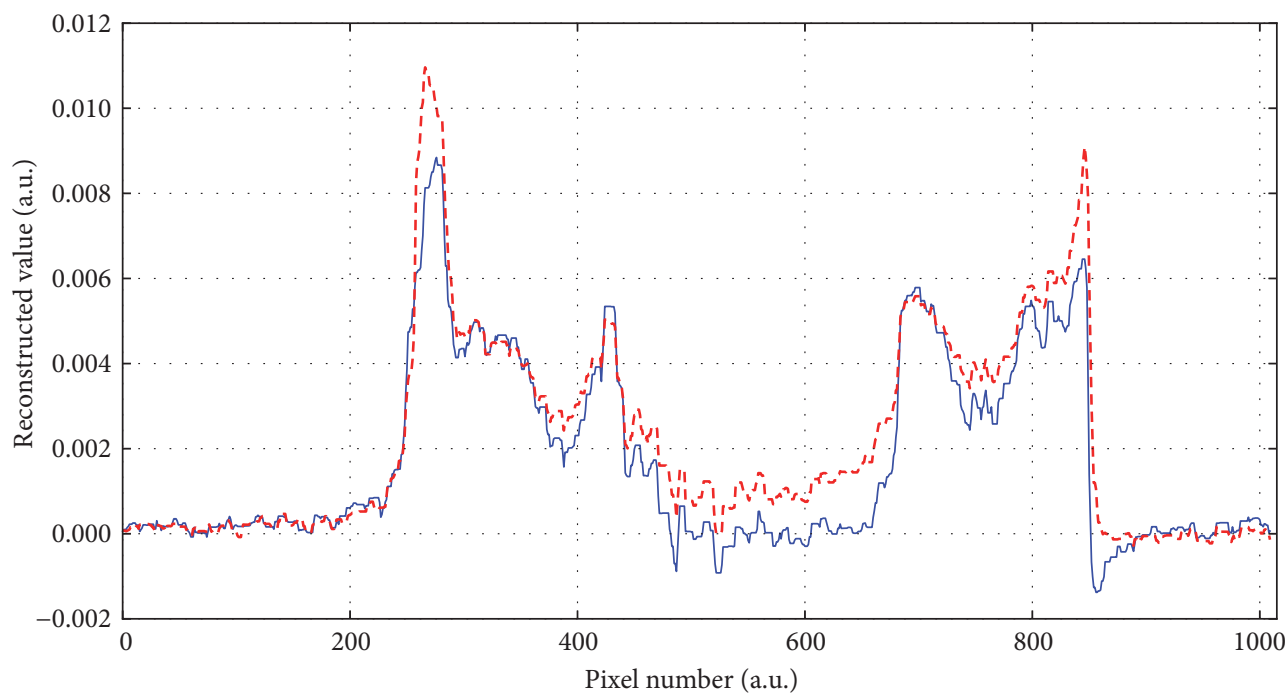

- Parameter is 1.83

- - - No correction

(c)

FIGURE 4: Reconstruction result: (a) without the $\mathrm{BH}$ correction, (b) with the $\mathrm{BH}$ correction, (c) the respective cross-sections on row 520.

of highly absorbing inclusions. With the found value, we corrected the projection data, thereby preparing its usage of the reconstruction algorithm.

We used the FBP algorithm to the projection data. The software implementation for python language is presented in the Astra Toolbox library [31].

Figures 4(a) and 5(a) show the reconstruction result without correction while Figures 4(b) and 5(b) show reconstruction after the suggested data linearization. Related crosssections are shown in Figures 4(c) and 5(c).

From the cross-section on row 520 (in Figure 4(c)), it can be seen that the hollow part inside the tooth has an absorption coefficient greater than the space outside the tooth in the reconstruction without correction (red line). Crosssection of the corrected data reconstruction (blue line) shows the absorption coefficient of the space outside the tooth, and the hollow part inside the tooth is the same; moreover, the absorption coefficient of the tooth itself remains unchanged.

It can be seen by cross-section on row 780 (in Figure 5(c)) that cupping effect artifact is brightly expressed in the reconstruction result without correction (red line). Crosssection of the corrected data reconstruction (blue line) shows that the cupping artifact completely suppressed, while the level of the absorption coefficient of the inner part remains unchanged.

We used the simulation results to test the procedure under high absorption conditions. We have calculated the spectrum of $100 \mathrm{kV}$ voltage $\mathrm{X}$-ray tube with $\mathrm{W}$ anode as a radiation source (Figure 6) by TASMIP Spectra Calculator [32].

Cross-section of the Fe phantom used to calculate the projections in parallel scheme is presented in Figure 7. 


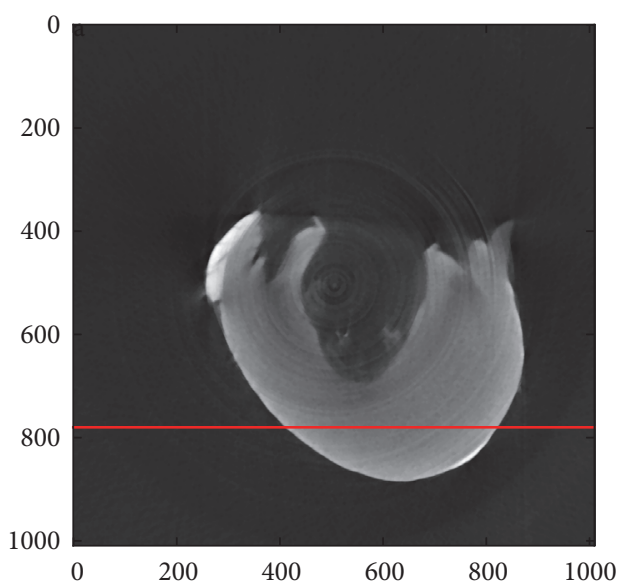

(a)

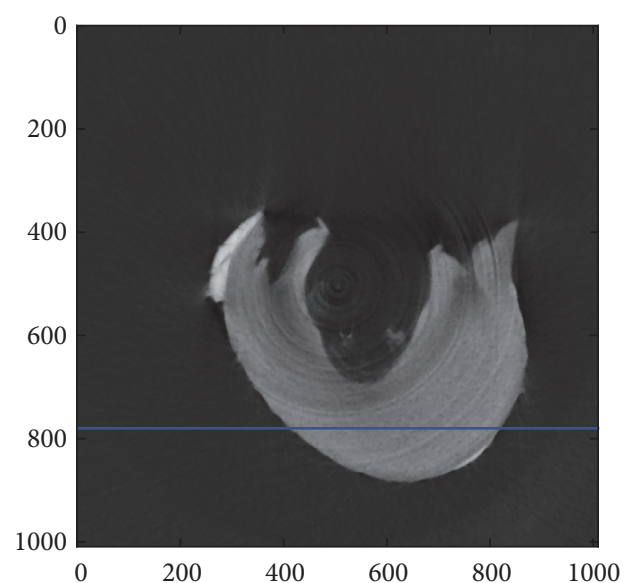

(b)

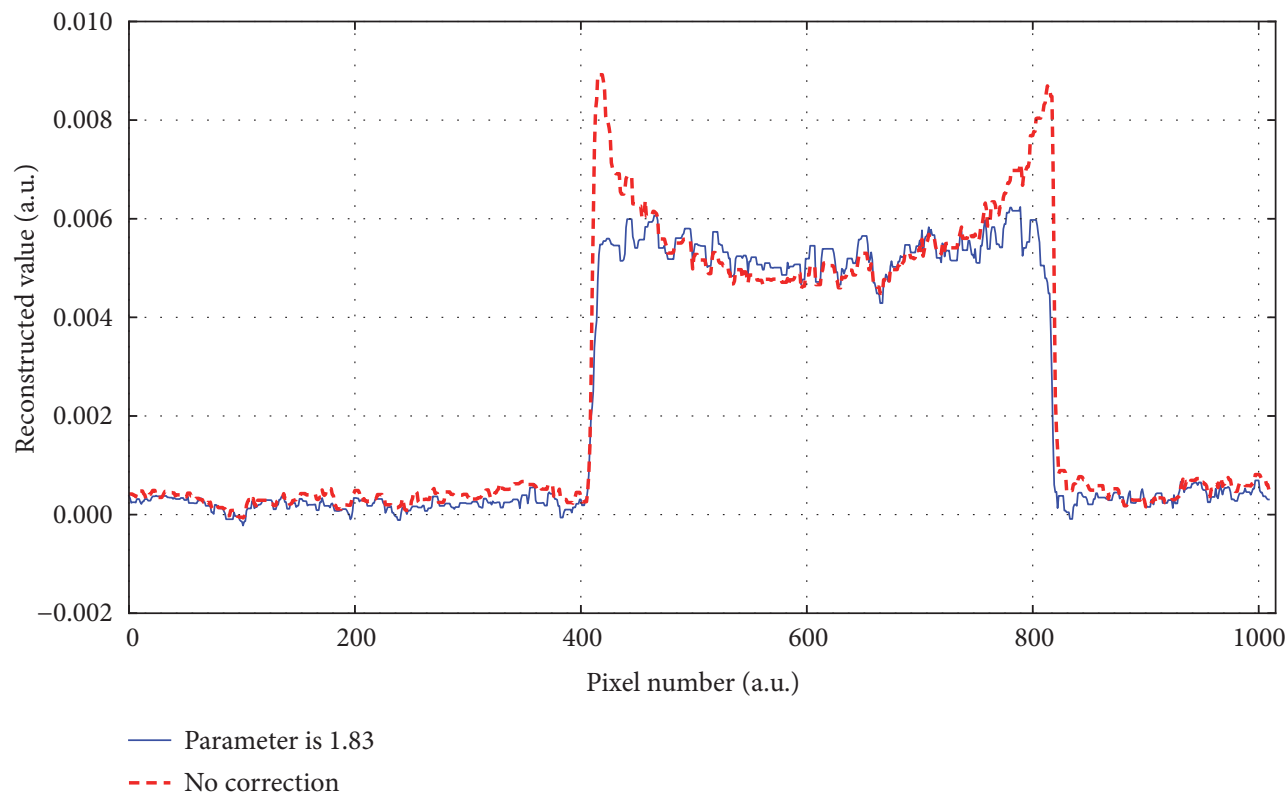

(c)

FIGURE 5: Reconstruction result: (a) without the BH correction, (b) with the $\mathrm{BH}$ correction, (c) the respective cross-sections on row 780.

The Astra Toolbox library [32] was used to calculate the projections.

Phantom size is 250 pixels. Pixel size is $28 \mu \mathrm{m}$. Number of evenly spaced projection angles is $180.1^{0}$ is a rotation angle value. Calculated sinogram is presented in Figure 8.

The behavior of criteria for different correction parameter values is presented in Figure 9. The minimum of functional (7) is achieved at 1.29.

We have used FBP for reconstruction. Reconstruction results are shown in Figure 10. Figure 10(a) presents results without linearization procedure. The reconstruction result obtained after linearization procedure with $\gamma=1.29$ is presented in Figure 10(b). Two 125-line cross-sections for both cases are presented in Figure 10(c).

After reliability inspection under high absorption conditions we used the procedure for data collected during the tomographic experiment. Steel alloy with nickel plated coating (test object) was scanned in cone beam geometry [33]. X-ray laboratory source with $\mathrm{W}$ anode was used. A source to object distance was $128.5 \mathrm{~mm}$ and source to detector distance was $228 \mathrm{~mm}$. Every rotational scan consisted of 360 projections with an angular constant step size of $1 \mathrm{deg}$. The detector pixel size was $0.055 \mathrm{~mm}$. Tomographic projection (top) and sinogram of central cross-section (bottom) are presented in Figure 11.

We applied the method of polychromatic data processing described above to the registered data. Figure 12 shows the behavior of criteria for different correction parameter values. It can be seen in Figure 12 that the optimal value of the parameter is 1.95 .

We have used FBP for reconstruction. Figure 13(a) presents the reconstruction result without correction while 


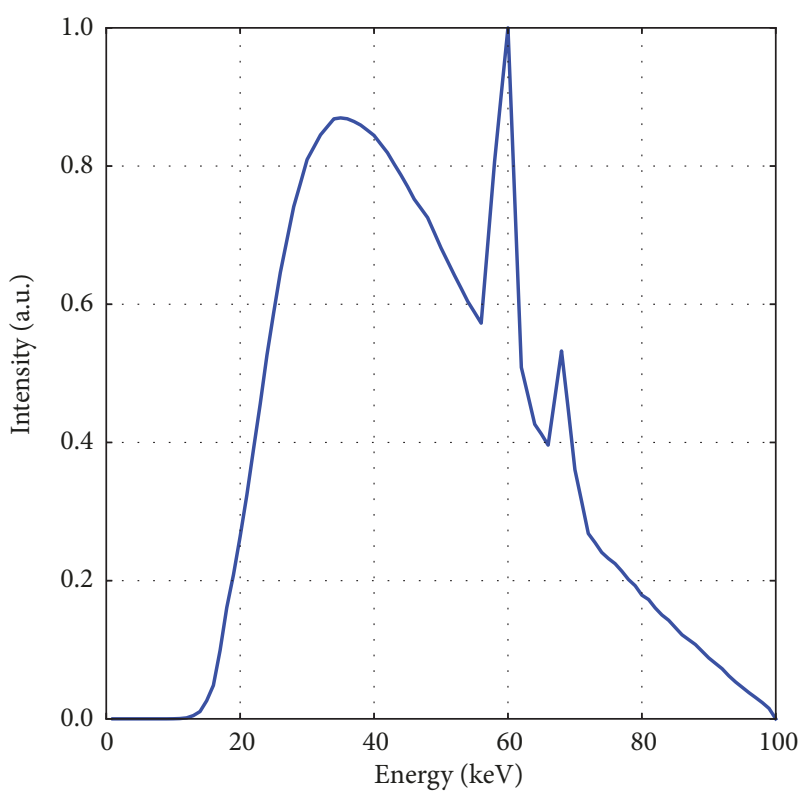

- W-anode, $\mathrm{x}$-ray spectra

FIgURE 6: Simulated X-ray spectra.

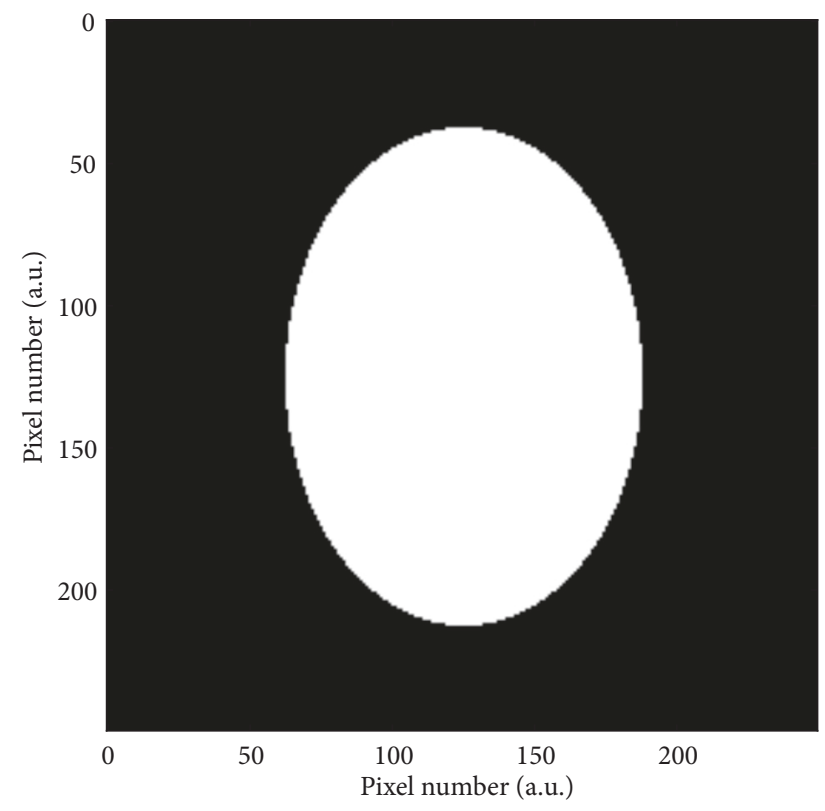

FIGURE 7: Cross-section of the Fe phantom.

Figure 13(b) shows reconstruction after the suggested data linearization. Related cross-sections are shown in Figure 13(c). As the object has nickel plated coating, two areas in Figure 13(c) illustrate the result of $\mathrm{BH}$ correction. The first one is near-boundary area (about 400 pixels and about 1200 pixels) and center area.

Ring inside the reconstructed area is explained by defects in some pixels of the used detector [34], which is created by the group that provided the data.

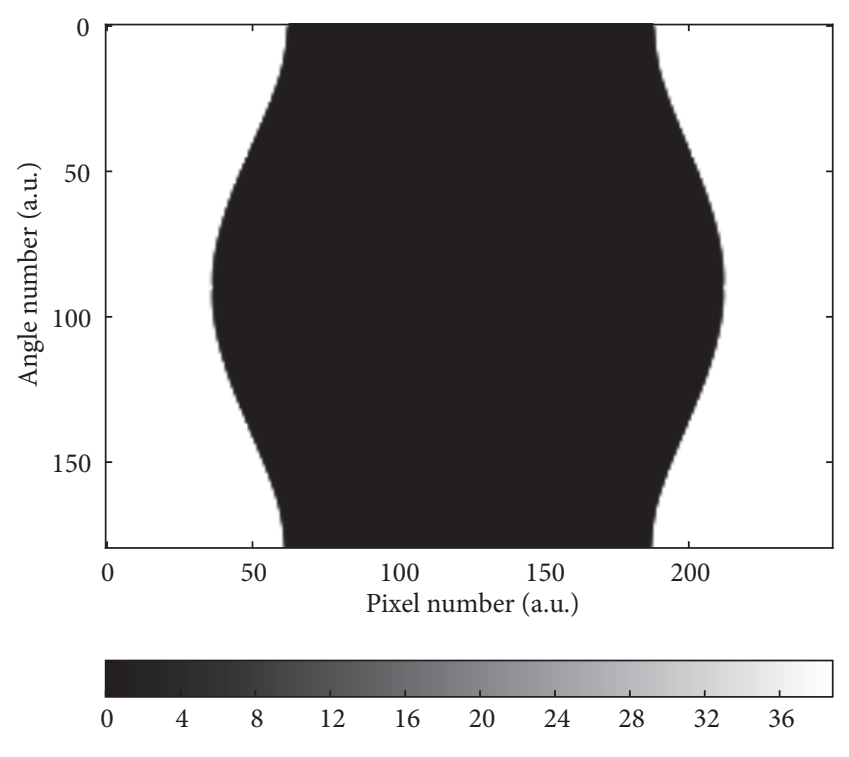

Figure 8: Phantom sinogram.

\section{Discussion}

To verify the optimality of the found value, we suggest the following analysis. We illustrate the steps of the analysis procedure using data from the baby's tooth as an example (Figure 5). With the first step, we select the region of interest (ROI) on the reconstructed image. The value inside ROI should be homogeneous (see the created ROI mask in Figure 14). With the second step, we calculate the standard deviation and mean value within the region of interest in the reconstruction result.

We made $2 \mathrm{D}$ reconstruction of the selected slice with parameters of the correction function in the range [1.0, 2.8]. Then we calculated the standard deviation in ROI. The results are shown in Figure 15. Red point in Figure 7 shows the standard deviation for the optimal parameter value.

The analysis shows that the found parameter value is optimal. Experiments with real data have confirmed that our approach is applicable. It should be noted that the suggested method is sensitive to distortions that violate the property of conservation of the Radon invariant. This group includes such distortions as the thermal drift of the center of the X-ray spot or a weak signal-to-noise ratio. On the other hand, the method is resistant to such artifacts as the displacement of the center of rotation.

The other way to evaluate the manifestation rate of the cupping artifact on the reconstructed ROI is to estimate the error based seminorm in the $W_{2}^{1}$ space:

$$
\left\|\mu_{f i t}\right\|_{W_{2}^{1}}=\left\{\int_{a}^{b} \mu_{f i t}^{\prime 2}(t) d t\right\}^{1 / 2}
$$

in ROI. To calculate the derivative we fitted the data to exclude salt-and-pepper noise from consideration. The colors used in Figure 16 correspond to the colors in Figure 5. To evaluate the potential gain of the cupping effect reduction, we set several 


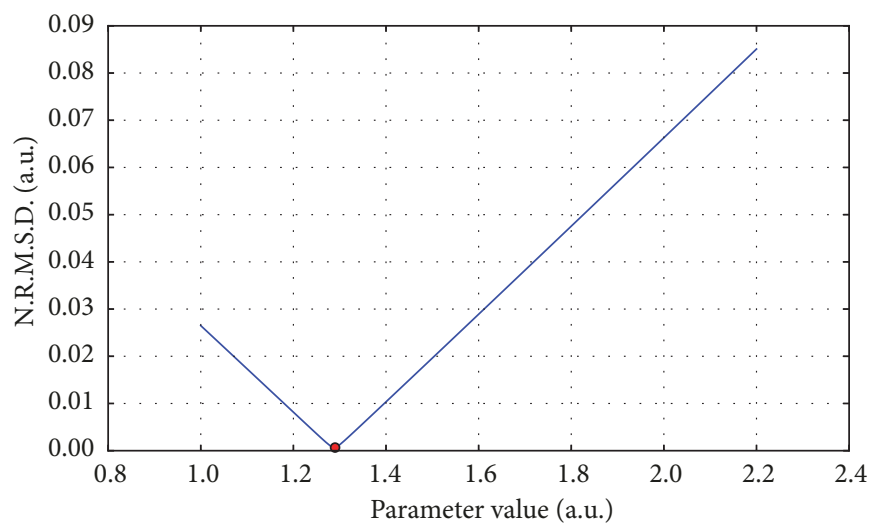

- N.R.M.S.D. dynamics

- Minimum value is 1.29

FIGURE 9: NRMSD dynamics depending on the parameter value.

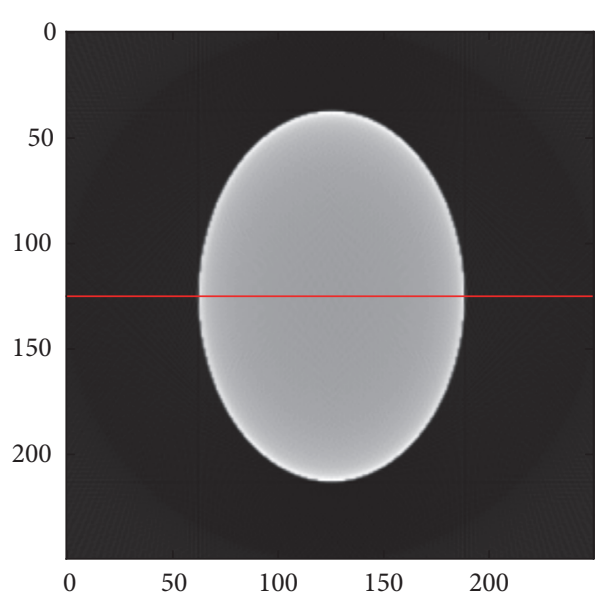

(a)

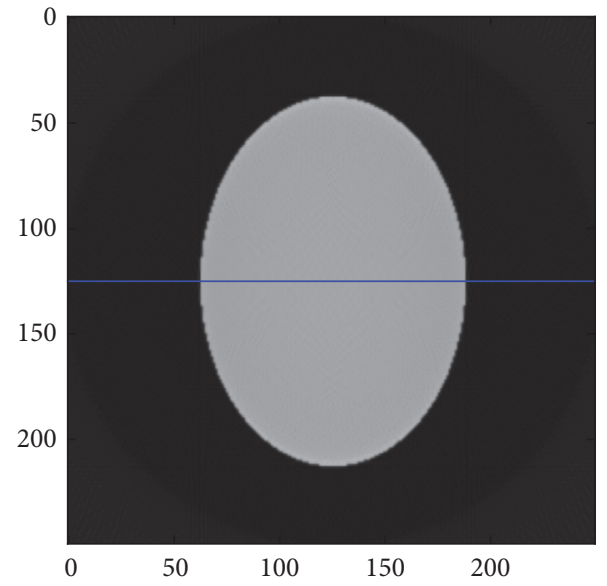

(b)

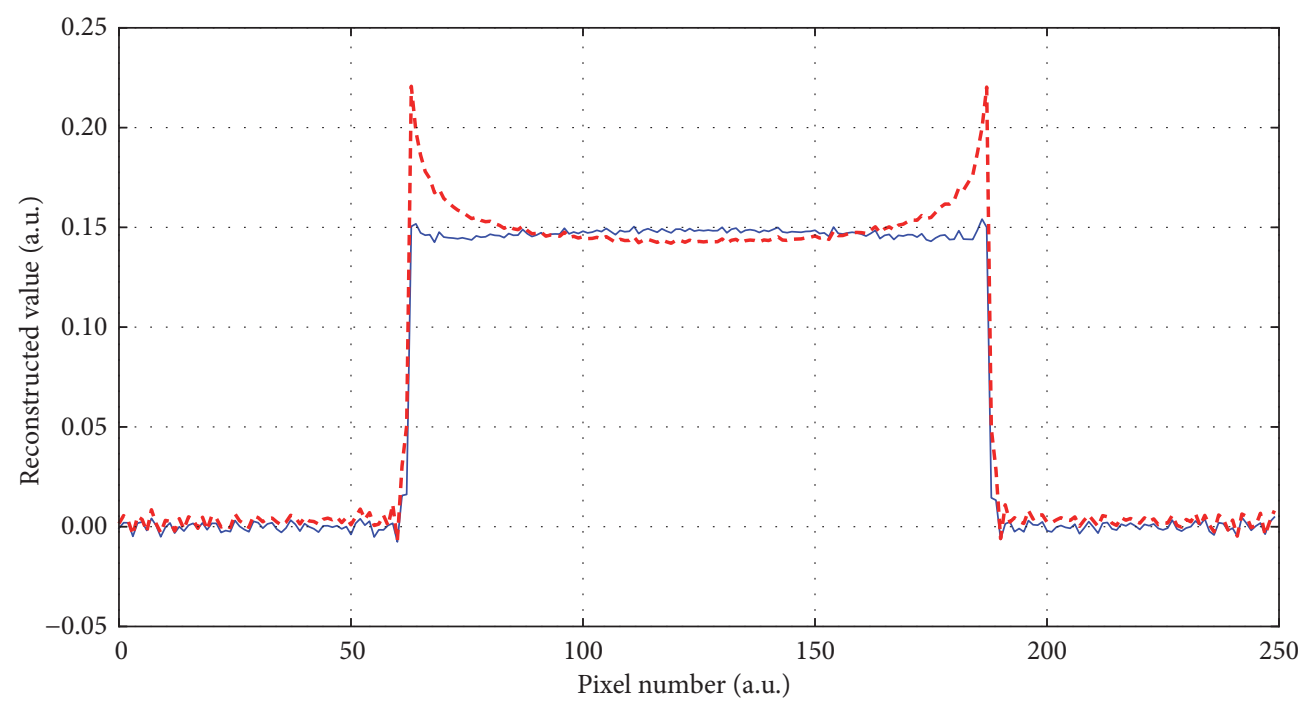

- Parameter is 1.29

- - - No correction

(c)

FIGURE 10: Reconstruction result: (a) without the BH correction, (b) with the BH correction, (c) the respective cross-sections on row 125. 

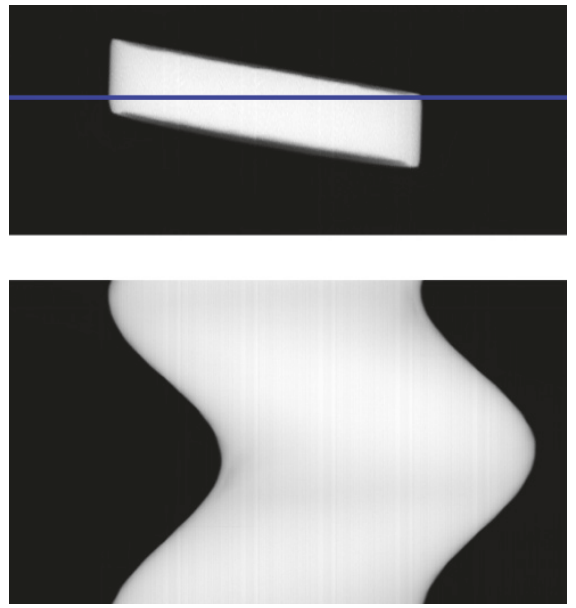

Figure 11: Projection (top) and sinogram (bottom) of the central cross-section.

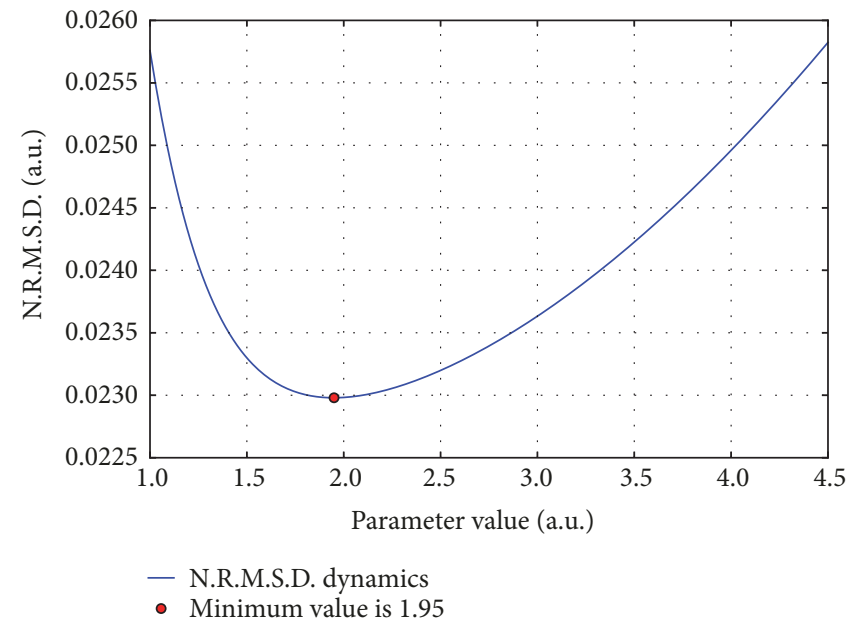

FIGURE 12: NRMSD dynamics depending on the parameter value.

different filter types (Figure 15) to substitute the standard ramp filter in FBP reconstruction [31].

Effect reduction of cupping artifact is around 3 times for each of them.

\section{Conclusions}

To demonstrate the decrease of the artifacts relevant to $\mathrm{BH}$, we apply the proposed algorithm to the data collected with the tomography set-up at FSRC "Crystallography and Photonics" RAS, data provided by our colleagues from Joint Institute for Nuclear Research, Dubna, and simulated data. First set of data are tomographic projections of baby's tooth. Object selection is driven by several reasons. The main reason is related to the fact that the presence of artifacts on CT images of teeth [35] is a serious problem when choosing a treatment regimen and when planning implantation procedures. The search is constantly in progress to find new methods to reduce artifacts. The second reason is due to the fact that the baby's tooth has a cavity formed by nonresorbed roots. It enabled us to evaluate the result of the algorithms operation both in the cross-section passing through the cavity (Figure 4) and in the cross-section passing through the dense part of the object. The second set is the projection data of test steel alloy with nickel plated coating. Its high absorption is a main reason to include the specimen in the consideration. The obtained results allow for the conclusion that the proposed approach, which does not require additional calibration experiments, allows reducing $\mathrm{BH}$ artifacts on the reconstructed image. We made reconstruction by FBP for data corrected with different power values to verify the developed system. The solution of the optimization problem gets in line with minimum STD position.

\section{Data Availability}

The data used to support the findings of this study are available from the corresponding author upon request. 


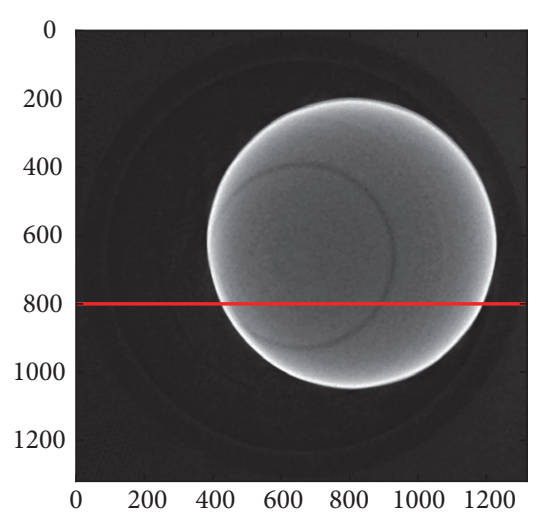

(a)

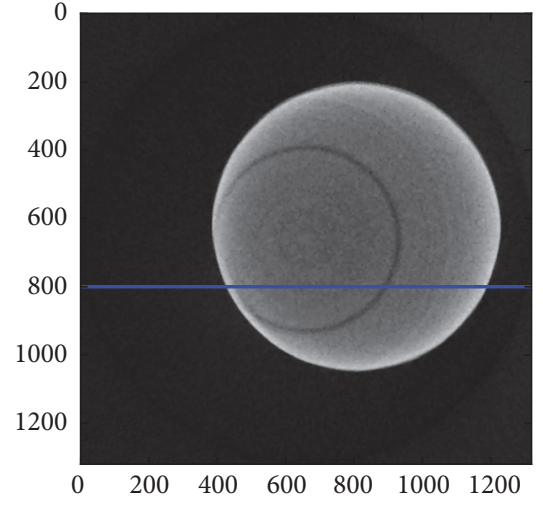

(b)

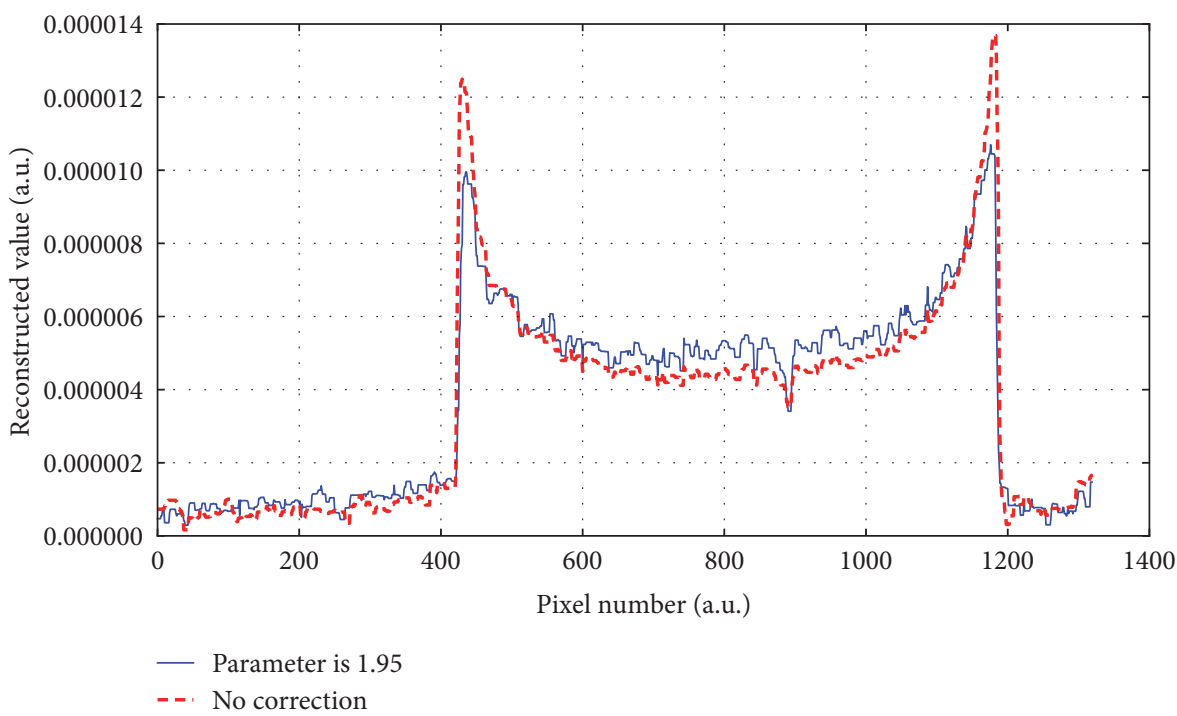

(c)

FIGURE 13: Reconstruction result: (a) without the $\mathrm{BH}$ correction, (b) with the $\mathrm{BH}$ correction, (c) the respective cross-sections on row 800.

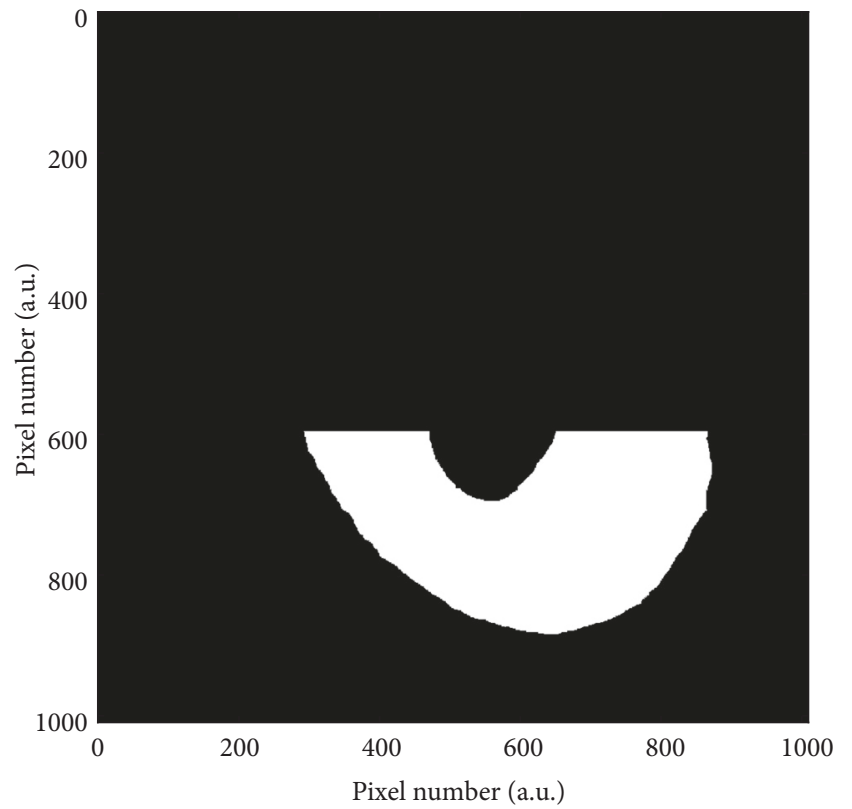

FIgURe 14: Mask.

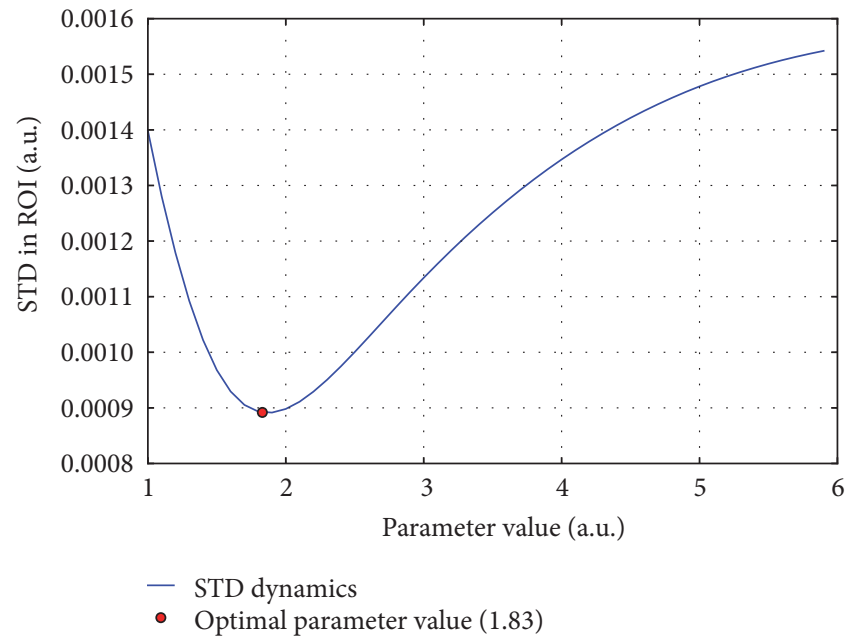

FIGURE 15: STD of reconstructed value in mask.

\section{Conflicts of Interest}

The authors declare that there are no conflicts of interest regarding the publication of this paper. 

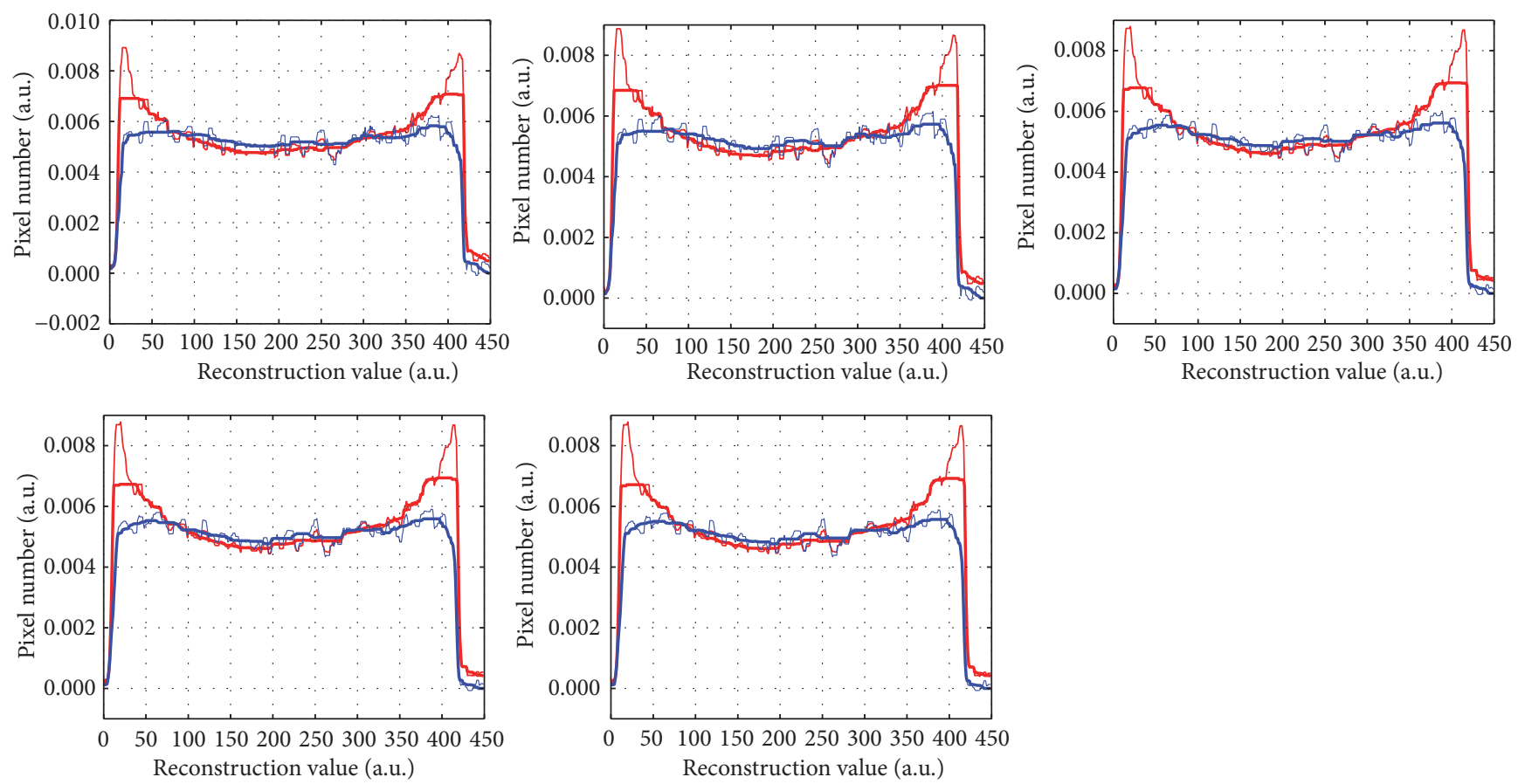

FIGURE 16: ROI for evaluation of the cupping artifact manifestation rate: (left-right, top-down) filters Hann, Hamming, Ram-Lak, SheppLogan, and cosine.

\section{Acknowledgments}

The authors thank Danila Kozhevnikov for the provided data. This work was supported by the Ministry of Science and Higher Education within the State assignment FSRC «Crystallography and Photonics» RAS in part of "tomography set-up developments" and Russian Foundation for Basic Research [project 18-29-26022] in part of "artifacts evaluation" and [project 17-29-03492] in part of "algorithm refinement".

\section{Supplementary Materials}

Supplementary materials include results that illustrate the output of the proposed method on model data. (Supplementary Materials)

\section{References}

[1] R. Wilk, "Application of Computed tomography and magnetic resonance in 3D modeling," in Stem Cells and Biomaterials for Regenerative Medicine, pp. 121-142, Academic Press, 2019.

[2] A. Nasim, R. Sasankoti Mohan, K. Nagaraju, S. Malik, S. Goel, and S. Gupta, "Application of cone beam computed tomography gray scale values in the diagnosis of cysts and tumors," Journal of Indian Academy of Oral Medicine \& Radiology, vol. 30, no. 1, p. 4, 2018.

[3] N. Drage, "Cone beam computed tomography (CBCT) in general dental practice," Primary dental journal, vol. 7, no. 1, pp. 26-30, 2018.

[4] M. M. Bornstein, K. Horner, and R. Jacobs, "Use of cone beam computed tomography in implant dentistry: current concepts, indications and limitations for clinical practice and research," Periodontology 2000, vol. 73, no. 1, pp. 51-72, 2017.

[5] L. De Chiffre, S. Carmignato, J.-P. Kruth, R. Schmitt, and A. Weckenmann, "Industrial applications of computed tomography," CIRP Annals - Manufacturing Technology, vol. 63, no. 2, pp. 655-677, 2014.

[6] A. Thompson, I. Maskery, and R. K. Leach, "X-ray computed tomography for additive manufacturing: a review," Measurement Science and Technology, vol. 27, no. 7, p. 072001, 2016.

[7] R. Gratton, J. Wilson, and K. Skuse, "Application of computed tomography for the examination of pressure retaining nuclear plant components," in Proceedings of the ASME 2017 Pressure Vessels and Piping Conference, p. V005T10A001, American Society of Mechanical Engineers, 2017.

[8] A. C. Kak and M. Slaney, Principles of Computerized Tomographic Imaging, IEEE Press, New York, NY, USA, 1988.

[9] J. Y. Huang, J. R. Kerns, J. L. Nute et al., "An evaluation of three commercially available metal artifact reduction methods for CT imaging," Physics in Medicine and Biology, vol. 60, no. 3, pp. 1047-1067, 2015.

[10] C. T. Dodge, E. P. Tamm, D. D. Cody et al., "Performance evaluation of iterative reconstruction algorithms for achieving CT radiation dose reduction - a phantom study," Journal of Applied Clinical Medical Physics, vol. 17, no. 2, pp. 511-531, 2016.

[11] R. Kasai, Y. Yamaguchi, T. Kojima, and T. Yoshinaga, “Tomographic image reconstruction based on minimization of symmetrized kullback-leibler divergence," Mathematical Problems in Engineering, vol. 2018, Article ID 8973131, 9 pages, 2018.

[12] K. Tateishi, Y. Yamaguchi, A. Al-Ola, Omar M., and T. Yoshinaga, "Continuous analog of accelerated OS-EM algorithm for computed tomography," Mathematical Problems in Engineering, vol. 2017, Article ID 1564123, 8 pages, 2017. 
[13] T. Kojima, T. Ueta, and T. Yoshinaga, "Multivalued discrete tomography using dynamical system that describes competition," Mathematical Problems in Engineering, vol. 2017, Article ID 8160354, 9 pages, 2017.

[14] J. P. Stonestrom, R. E. Alvarez, and A. Macovski, "A framework for spectral artifact corrections in X-ray CT," IEEE Transactions on Biomedical Engineering, vol. 2, pp. 128-141, 1981.

[15] R. A. Brooks and G. Di Chiro, "Beam hardening in X-ray reconstructive tomography," Physics in Medicine and Biology, vol. 21, no. 3, pp. 390-398, 1976.

[16] R. J. Jennings, "A method for comparing beam-hardening filter materials for diagnostic radiology," Medical Physics, vol. 15, no. 4, pp. 588-599, 1988.

[17] S. Chen, X. Xi, L. Li et al., "A filter design method for beam hardening correction in middle-energy $\mathrm{x}$-ray computed tomography," in Eighth International Conference on Digital Image Processing (ICDIP 2016), vol. 10033, p. 1003352, International Society for Optics and Photonics, 2016.

[18] A. J. Coleman and M. Sinclair, "A beam-hardening correction using dual-energy computed tomography," Physics in Medicine and Biology, vol. 30, no. 11, pp. 1251-1256, 1985.

[19] M. van Geet, R. Swennen, and M. Wevers, "Quantitative analysis of reservoir rocks by microfocus X-ray computerised tomography," Sedimentary Geology, vol. 132, no. 1-2, pp. 25-36, 2000.

[20] L. Yu, S. Leng, and C. H. McCollough, "Dual-energy CTbased monochromatic imaging," AJR. American Journal of Roentgenology, vol. 199 (5_supplement), pp. S9-S15, 2012.

[21] G. T. Herman, "Correction for beam hardening in computed tomography," Physics in Medicine and Biology, vol. 24, no. 1, pp. 81-106, 1979.

[22] P. Hammersberg and M. Mångård, "Correction for beam hardening artefacts in computerised tomography," Journal of XRay Science and Technology, vol. 8, no. 1, pp. 75-93, 1998.

[23] L. Brabant, E. Pauwels, M. Dierick, D. Van Loo, M. A. Boone, and L. Van Hoorebeke, "A novel beam hardening correction method requiring no prior knowledge, incorporated in an iterative reconstruction algorithm," NDT \& E International, vol. 51, pp. 68-73, 2012.

[24] P. Jin, C. A. Bouman, and K. D. Sauer, "A model-based image reconstruction algorithm with simultaneous beam hardening correction for X-ray CT,' IEEE Transactions on Computational Imaging, vol. 1, no. 3, pp. 200-216, 2015.

[25] J. Hsieh, R. C. Molthen, C. A. Dawson, and R. H. Johnson, “An iterative approach to the beam hardening correction in cone beam CT”, Medical Physics, vol. 27, no. 1, pp. 23-29, 2000.

[26] S. Xie, W. Zhuang, B. Li, P. Bai, W. Shao, and Y. Tong, "Blind deconvolution combined with level set method for correcting cupping artifacts in cone beam CT," in Medical Imaging 2017: Image Processing, vol. 10133, p. 101331Z, International Society for Optics and Photonics.

[27] S. Sarkar, P. Wahi, and P. Munshi, "An empirical correction method for beam-hardening artifact in Computerized Tomography (CT) images," NDT \& E International, vol. 102, pp. 104113, 2019.

[28] M. Chukalina, A. Ingacheva, A. Buzmakov et al., "Automatic beam hardening correction for CT reconstruction," in Proceedings of the 31st European Conference on Modelling and Simulation, ECMS 2017, pp. 270-275, Budapest, Hungary, May 2017.
[29] A. Buzmakov, M. Chukalina, D. Nikolaev et al., "Monochromatic computed microtomography using laboratory and synchrotron sources and X-ray fluorescence analysis for comprehensive analysis of structural changes in bones," Journal of Applied Crystallography, vol. 48, no. 3, pp. 693-701, 2015.

[30] K. Mueller, Fast and accurate three-dimensional reconstrution from cone-beam projection data using algebraic methods [Doctoral thesis], The Ohio State University, 1998.

[31] W. van Aarle, W. J. Palenstijn, J. Cant et al., "Fast and flexible Xray tomography using the ASTRA toolbox," Optics Express, vol. 24, no. 22, pp. 25129-25147, 2016.

[32] J. Punnoose, J. Xu, A. Sisniega, W. Zbijewski, and J. H. Siewerdsen, "Technical note: spektr 3.0-A computational tool for x-ray spectrum modeling and analysis," Medical Physics, vol. 43, no. 8, pp. 4711-4717, 2016.

[33] A. Gongadze, A. Zhemchugov, G. Chelkov et al., "Alignment and resolution studies of a MARS CT scanner," Physics of Particles and Nuclei Letters, vol. 12, no. 5, pp. 725-735, 2015.

[34] D. Kozhevnikov and P. Smolyanskiy, "Equalization of Medipix family detector energy thresholds using X-ray tube spectrum high energy cut-off," Journal of Instrumentation, vol. 14, no. 01, pp. T01006-T01006, 2019.

[35] K. F. Vasconcelos, F. P. Nicolielo, M. C. Nascimento et al., "Artefact expression associated with several cone-beam computed tomographic machines when imaging root filled teeth," International Endodontic Journal, vol. 48, pp. 994-1000, 2015. 


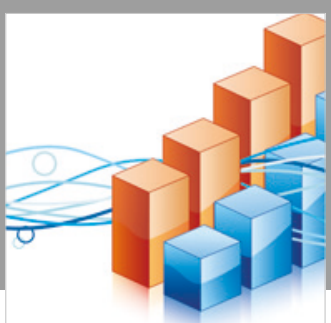

Advances in

Operations Research

\section{-n-m}
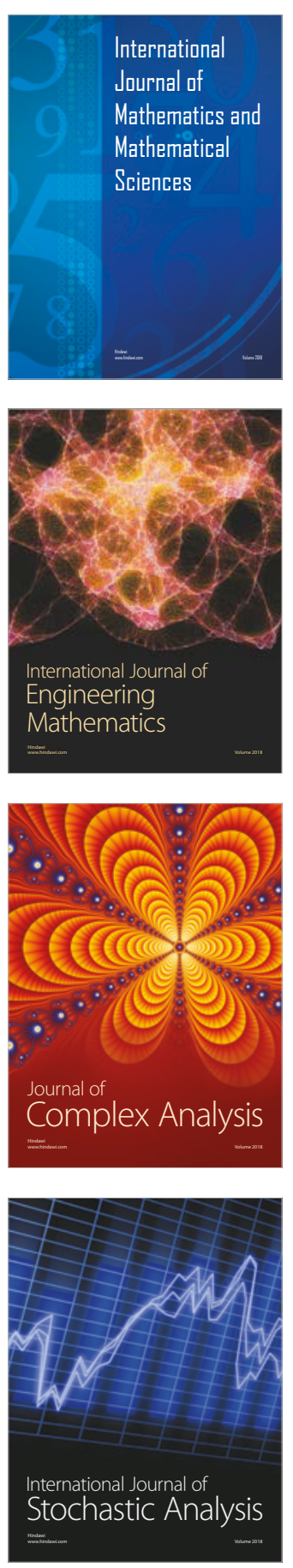
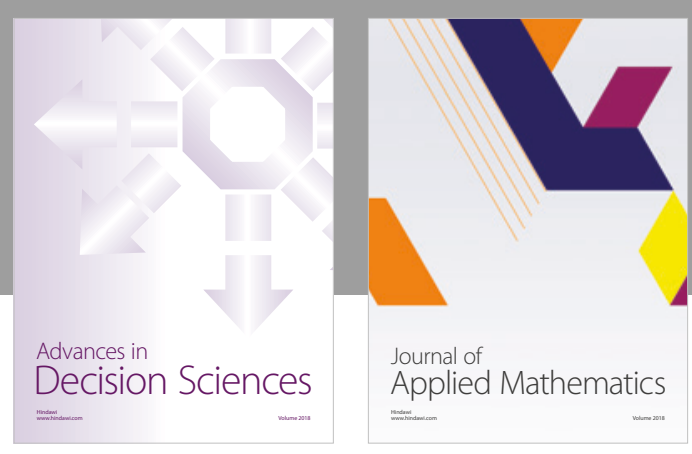

Journal of

Applied Mathematics
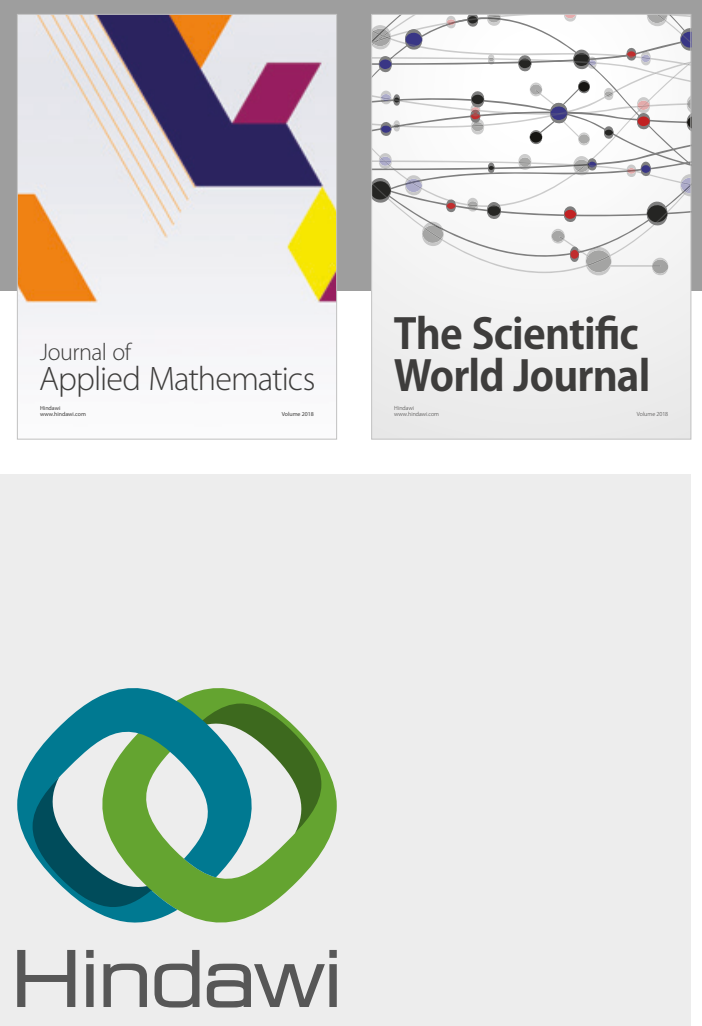

Submit your manuscripts at

www.hindawi.com

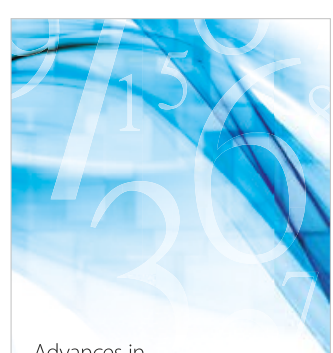

Advances in
Numerical Analysis
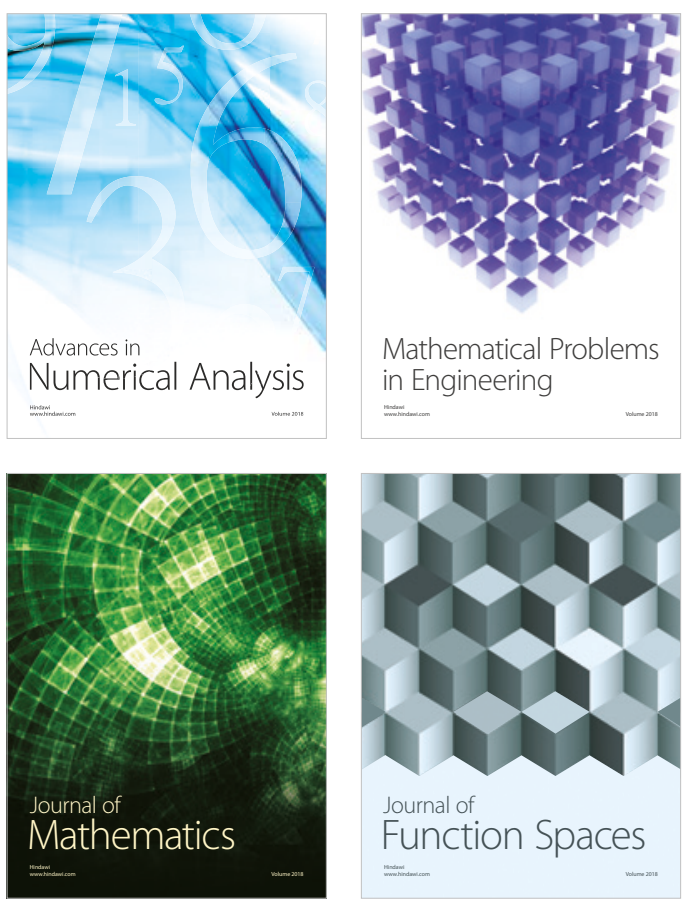

Mathematical Problems in Engineering

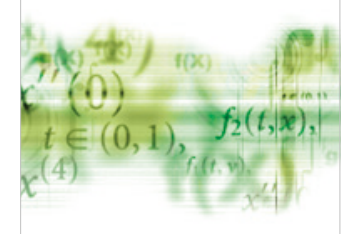

International Journal of

Differential Equations

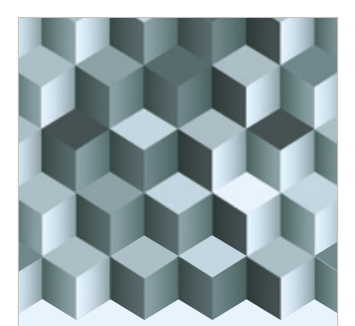

Journal of

Function Spaces

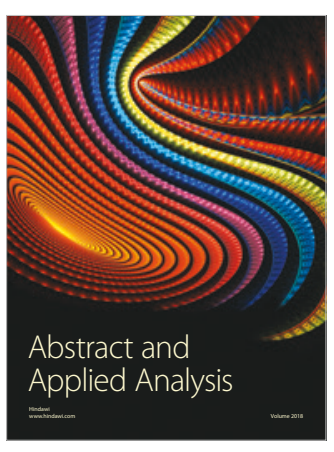

The Scientific

World Journal

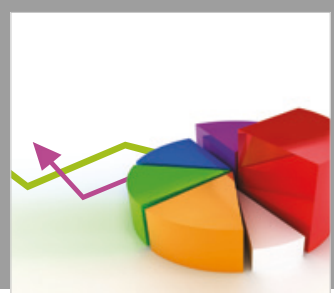

Journal of

Probability and Statistics
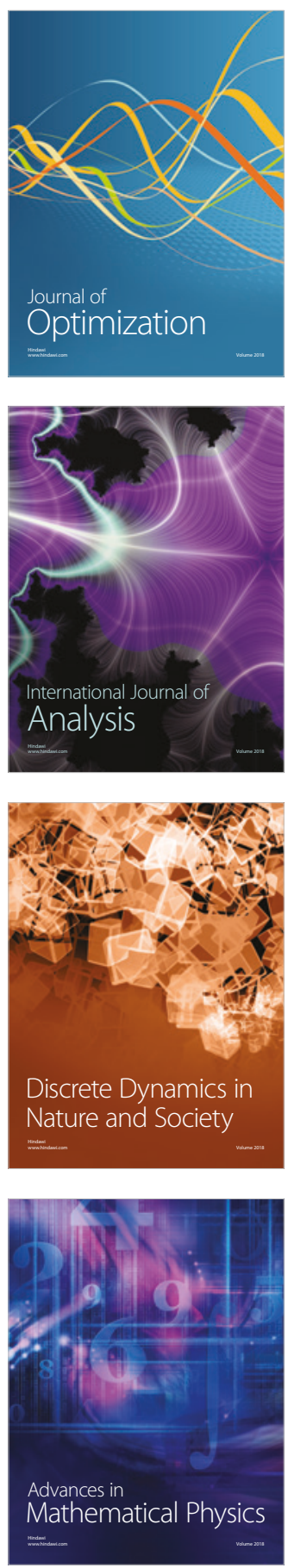